\title{
STRONG CONVERGENCE OF APPROXIMATING FIXED POINT SEQUENCES FOR NONEXPANSIVE MAPPINGS
}

\author{
HONG-Kun Xu
}

\begin{abstract}
Consider a nonexpansive self-mapping $T$ of a bounded closed convex subset of a Banach space. Banach's contraction principle guarantees the existence of approximating fixed point sequences for $T$. However such sequences may not be strongly convergent, in general, even in a Hilbert space. It is shown in this paper that in a real smooth and uniformly convex Banach space, appropriately constructed approximating fixed point sequences can be strongly convergent.
\end{abstract}

\section{INTRODUCTION}

Let $X$ be a real Banach space and $C$ be a closed convex subset of $X$. Let $T: C \rightarrow C$ be a self-mapping of $C$. Recall that $T$ is said to be nonexpansive if

$$
\|T x-T y\| \leqslant\|x-y\|
$$

for all $x, y \in C$. We use $\operatorname{Fix}(T)$ to denote the set of fixed points of $T$ (that is, $\operatorname{Fix}(T)$ $=\{x \in C: T x=x\})$. Throughout this article, we assume that $\operatorname{Fix}(T)$ is nonempty.

Recall also that a sequence $\left\{x_{n}\right\}$ in $C$ is said to be an approximating fixed point sequence for $T$ if

$$
\lim _{n \rightarrow \infty}\left\|x_{n}-T x_{n}\right\|=0 .
$$

There are several ways to construct an approximating fixed point sequence for a nonexpansive mapping $T$. We mention two below.

Firstly we can use Banach's contraction principle to obtain a sequence $\left\{x_{n}\right\}$ in $C$ such that

$$
x_{n}=t_{n} x_{0}+\left(1-t_{n}\right) T x_{n}, \quad n \geqslant 1
$$

where the initial guess $x_{0}$ is taken arbitrarily in $C$ and $\left\{t_{n}\right\}$ is a sequence in the interval $(0,1)$ such that $t_{n} \rightarrow 0$ as $n \rightarrow \infty$. Due to the assumption that $\operatorname{Fix}(T) \neq \emptyset$, this sequence $\left\{x_{n}\right\}$ is bounded (indeed $\left\|x_{n}-p\right\| \leqslant\left\|x_{0}-p\right\|$ for all $p \in \operatorname{Fix}(T)$ ). Hence

$$
\left\|x_{n}-T x_{n}\right\|=t_{n}\left\|x_{0}-T x_{n}\right\| \rightarrow 0
$$

Received 26th April, 2006

Supported in part by the National Research Foundation of South Africa.

Copyright Clearance Centre, Inc. Serial-fee code: 0004-9727/06 \$A2.00+0.00. 
and $\left\{x_{n}\right\}$ is an aproximating fixed point sequence for $T$.

Secondly, we use Mann's iteration process [8] to generate a sequence $\left\{x_{n}\right\}$ in $C$ by the recursive formula

$$
x_{n+1}=\left(1-\alpha_{n}\right) x_{n}+\alpha_{n} T x_{n}, \quad n \geqslant 0
$$

where the initial guess $x_{0} \in C$ is arbitrary, and the sequence $\left\{\alpha_{n}\right\}$ lies in the interval $(0,1)$. This sequence $\left\{x_{n}\right\}$ is bounded since, for any $p \in \operatorname{Fix}(T)$, we have

$$
\left\|x_{n+1}-p\right\| \leqslant\left(1-\alpha_{n}\right)\left\|x_{n}-p\right\|+\alpha_{n}\left\|T x_{n}-p\right\| \leqslant\left\|x_{n}-p\right\| .
$$

That is, $\left\{\left\|x_{n}-p\right\|\right\}$ is a nonincreasing sequence. Moreover, it is not hard to find that the sequence $\left\{\left\|x_{n}-T x_{n}\right\|\right\}$ is also nonincreasing; hence $\lim _{n}\left\|x_{n}-T x_{n}\right\|$ exists.

However, it is not known whether this sequence $\left\{x_{n}\right\}$ is always an approximating fixed point sequence of $T$. Only partial answers have been obtained. Indeed, if the space $X$ is uniformly convex and if the control sequence $\left\{\alpha_{n}\right\}$ satisfies the condition $\sum_{n=0}^{\infty} \alpha_{n}\left(1-\alpha_{n}\right)=\infty$, then Reich [12] showed that the sequence $\left\{x_{n}\right\}$ generated by Mann's iteration process (1.1) is an approximating fixed point sequence of $T$. For the sake of completeness, we include a brief proof to this fact. Let $\delta_{X}$ be the modulus of convexity of $X$. Pick a $p \in \operatorname{Fix}(T)$. Assuming $\left\|x_{n}-p\right\|>0$ and noticing $\left\|T x_{n}-p\right\| \leqslant\left\|x_{n}-p\right\|$, we deduce that

$$
\left\|x_{n+1}-p\right\| \leqslant\left\|x_{n}-p\right\|\left[1-2 \alpha_{n}\left(1-\alpha_{n}\right) \delta_{X}\left(\frac{\left\|x_{n}-T x_{n}\right\|}{\left\|x_{n}-p\right\|}\right)\right] .
$$

Hence

$$
\sum_{n=0}^{\infty} \alpha_{n}\left(1-\alpha_{n}\right)\left\|x_{n}-p\right\| \delta_{X}\left(\frac{\left\|x_{n}-T x_{n}\right\|}{\left\|x_{n}-p\right\|}\right) \leqslant\left\|x_{0}-p\right\|<\infty
$$

Put $r=\lim _{n}\left\|x_{n}-p\right\|$. If $r=0$, we are done. So assume $r>0$. If $\sum_{n=0}^{\infty} \alpha_{n}\left(1-\alpha_{n}\right)=\infty$, we obtain from (1.2) that $\lim _{n} \delta_{X}\left(\left\|x_{n}-T x_{n}\right\| / r\right)=0$. This implies that $\lim _{n}\left\|x_{n}-T x_{n}\right\|=0$ and $\left\{x_{n}\right\}$ is an approximating fixed point sequence of $T$.

An approximating fixed point sequence is not necessarily always weakly convergent though it is true that in a Hilbert space every weak limit point of an approximating fixed point sequence is always a fixed point of $T$. This fact is called the demiclosedness principle for nonexpansive mappings which indeed holds in uniformly convex Banach spaces as stated in the next lemma.

LEMma 1.1. (See [4].) Let $X$ be a uniformly convex Banach space, $C$ a closed convex subset of $C$, and $T: C \rightarrow C$ a nonexpansive mapping with a fixed point. Then $I-T$ is demiclosed in the sense that if $\left\{x_{n}\right\}$ is a sequence in $C$ and if $x_{n} \rightarrow x$ weakly and $(I-T) x_{n} \rightarrow y$ strongly for some $x$ and $y$, then $(I-T) x=y$. 
In a summary, in the setting of real uniformly convex Banach spaces $X$, what is clear is that every weak limit point of an approximating fixed point sequence for $T$ is a fixed point of $T$. However it remains unclear if the entire approximating fixed point sequence is weakly convergent. Reich [12] proves that if, in addition, $X$ also has a Frechet differentiable norm and if $\left\{x_{n}\right\}$ is an approximating fixed point sequence generated by Mann's iteration process $(1.1)$, then $\left\{x_{n}\right\}$ is weakly convergent.

In general, an approximating fixed point sequence may fail to be strongly convergent even in the Hilbert space setting [3].

It is the purpose of this note to prove that an appropriately constructed approximating fixed point sequence can be strongly convergent in a smooth and uniformly convex Banach space. For more recent investigations on strong convergence for nonexpansive and maximal monotone mappings, see $[5,6,7,9,10,11,13,14,15,17]$ and the references therein.

\section{Projections in Uniformly CONVEX Banach SPACES}

Let $X$ be a real uniformly convex Banach space $X$. Thus, for every $\varepsilon>0, \delta_{X}(\varepsilon)>0$, where $\delta_{X}$ is the modulus of convexity of $X$ defined by

$$
\delta_{X}(\varepsilon)=\inf \left\{1-\frac{1}{2}\|x+y\|:\|x\| \leqslant 1,\|y\| \leqslant 1,\|x-y\| \geqslant \varepsilon\right\} .
$$

Let $C$ be a nonempty closed convex subset of $X$. Like the Hilbert space case, we can define the nearest point projection $P_{C}$ from $X$ onto $C$ by assigning to each $x \in X$ the only point $P_{C} x$ in $C$ with the property

$$
\left\|x-P_{C} x\right\|=\inf \{\|x-y\|: y \in C\} .
$$

This projection $P_{C}$, though continuous (indeed uniformly continuous on bounded sets), is however inconvenient to use because it is not nonexpansive anymore (hence $I-P_{C}$ lacks monotonicity), as contrast to the nonexpansivity of nearest point projections in a Hilbert space. Instead, another kind of projections has been introduced to replace the nearest point projections, which is however still denoted by the same notation $P_{C}$. That is, in the rest of the paper, by $P_{C}$ we mean the projection from $X$ onto $C$ introduced as follows.

Let $J: X \rightarrow X^{*}$ be the duality map of $X$ defined by

$$
J(x)=\left\{x^{*} \in X^{*}:\left\langle x, x^{*}\right\rangle=\|x\|^{2}=\left\|x^{*}\right\|^{2}\right\}, \quad x \in X .
$$

Assume $X$ is smooth so that $J$ is single-valued on $X$ and hence we can define a function $\varphi$ on $X \times X$ by (see $[\mathbf{1}, \mathbf{5}])$

$$
\varphi(x, y)=\|x\|^{2}-2\langle x, J(y)\rangle+\|y\|^{2}, \quad x, y \in X .
$$


It is easily seen that

$$
(\|x\|-\|y\|)^{2} \leqslant \varphi(x, y) \leqslant(\|x\|+\|y\|)^{2}, \quad x, y \in X .
$$

Since for each fixed $y, \varphi(\cdot, y)$ is a continuous strictly convex function on $X$, there is a unique point $z \in C$ which solves the minimisation

$$
\varphi(z, y)=\min \{\varphi(x, y): x \in C\} .
$$

This unique point $z$ in $C$ is called the (generalised) projection of $y$ onto $C$. That is, we define the projection operator $P_{C}: X \rightarrow C$ by setting

$$
P_{C} y=z,
$$

where $z$ is the only point in $C$ satisfying (2.2). (Note that if $X$ is a Hilbert space, $\varphi(x, y)=\|x-y\|^{2}$. Hence the projection $P_{C}$ defined in (2.3) coincides with the nearest point projection onto $C$ in the Hilbert space setting.)

The next proposition gathers some basic properties of $P_{C}$ which will be used in the proof of the main result in the next section.

PROPOSITION 2.1. Assume that $X$ is a smooth and uniformly convex Banach space and $C$ is a nonempty closed convex subset of $X$.

(i) Given sequences $\left\{x_{n}\right\}$ and $\left\{y_{n}\right\}$ in $X$. If one of them is bounded, then $\varphi\left(x_{n}, y_{n}\right) \rightarrow 0$ if and only if $\left\|x_{n}-y_{n}\right\| \rightarrow 0$.

(ii) Given $y \in X$ and $z \in C$. Then $z=P_{C} y$ if and only if there holds the inequality:

$$
\langle v-z, J(z)-J(y)\rangle \geqslant 0 \quad \forall v \in C .
$$

(iii) The following inequality holds:

$$
\varphi\left(x, P_{C} y\right)+\varphi\left(P_{C} y, y\right) \leqslant \varphi(x, y) \quad \forall x \in C, y \in X .
$$

Proof: (i) The necessity part is proved in [5] under the stronger condition that the space $X$ be uniformly smooth. The uniform smoothness can be indeed weakened to smoothness. To see this, we notice that if $\varphi\left(x_{n}, y_{n}\right) \rightarrow 0$ and if one of the sequences $\left\{x_{n}\right\}$ and $\left\{y_{n}\right\}$ is bounded, then both $\left\{x_{n}\right\}$ and $\left\{y_{n}\right\}$ are bounded. Let $r>0$ be such that the closed ball $B_{r}=\{u \in X:\|u\| \leqslant r\}$ contains all the points of $\left\{x_{n}\right\},\left\{y_{n}\right\}$ and $\left\{x_{n}-y_{n}\right\}$. By Xu [16], we have a continuous strictly increasing function $g:[0, \infty) \rightarrow[0, \infty)$ with $g(0)=0$ and satisfying the property:

$$
\|u+v\|^{2} \geqslant\|u\|^{2}+2\langle v, J(u)\rangle+g(\|v\|), \quad \forall u, v \in B_{r} .
$$


In particular,

$$
\begin{aligned}
\left\|x_{n}\right\|^{2} & =\left\|y_{n}+\left(x_{n}-y_{n}\right)\right\|^{2} \\
& \geqslant\left\|y_{n}\right\|^{2}+2\left\langle x_{n}-y_{n}, J\left(y_{n}\right)\right\rangle+g\left(\left\|x_{n}-y_{n}\right\|\right) \\
& =-\left\|y_{n}\right\|^{2}+2\left\langle x_{n}, J\left(y_{n}\right)\right\rangle+g\left(\left\|x_{n}-y_{n}\right\|\right) .
\end{aligned}
$$

It now follows from the definition of $\varphi$ that

$$
g\left(\left\|x_{n}-y_{n}\right\|\right) \leqslant \varphi\left(x_{n}, y_{n}\right) \rightarrow 0 .
$$

Therefore $\left\|x_{n}-y_{n}\right\| \rightarrow 0$.

To see the sufficiency part (true indeed in any smooth Banach space), we assume $\left\|x_{n}-y_{n}\right\| \rightarrow 0$ and thus both sequences $\left\{x_{n}\right\}$ and $\left\{y_{n}\right\}$ are bounded. That $\varphi\left(x_{n}, y_{n}\right) \rightarrow 0$ now follows from the following computations:

$$
\begin{aligned}
\varphi\left(x_{n}, y_{n}\right) & =\left\|x_{n}\right\|^{2}-\left\|y_{n}\right\|^{2}-2\left\langle x_{n}-y_{n}, J\left(y_{n}\right)\right\rangle \\
& \leqslant\left\|x_{n}-y_{n}\right\|\left(\left\|x_{n}\right\|+3\left\|y_{n}\right\|\right) .
\end{aligned}
$$

(ii) Since for each fixed $y \in X, \varphi(\cdot, y)$ is convex, $z \in C$ is a minimiser of $\varphi(\cdot, y)$ over $C$ if and only if there holds the optimality condition:

$$
\langle\nabla \varphi(z, y), v-z\rangle \geqslant 0 \quad \forall v \in C
$$

where $\nabla \varphi(z, y)$ is the gradient of $\varphi(\cdot, y)$ at $z$. Since it is easily computed that

$$
\langle\nabla \varphi(z, y), v-z\rangle=2\langle v-z, J(z)-J(y)\rangle
$$

we obtain (2.4).

(iii) Using the definition of $\varphi$, we find that (2.5) is equivalent to the inequality:

$$
\left\langle P_{C} y-x, J\left(P_{C} y\right)-J(y)\right\rangle \leqslant 0 .
$$

This is however the inequality (2.4) with $v$ and $z$ replaced by $x$ and $P_{C} y$, respectively.

We shall use the notation:

1. $\rightarrow$ for weak convergence and $\rightarrow$ for strong convergence.

2. $\omega_{w}\left(x_{n}\right)=\left\{x: \exists x_{n_{j}} \rightarrow x\right\}$ denotes the weak $\omega$-limit set of $\left\{x_{n}\right\}$.

LEMMA 2.2. Let $X$ be a real smooth and uniformly convex Banach space and $K$ be a nonempty closed convex subset of $X$. Let $\left\{x_{n}\right\}$ be a bounded sequence in $X$ and $u \in X$. Let $q=P_{K} u$. Assume that $\left\{x_{n}\right\}$ satisfies the conditions

(i) $\omega_{w}\left(x_{n}\right) \subset K$ and

(ii) $\varphi\left(x_{n}, u\right) \leqslant \varphi(q, u)$ for all $n$.

Then $x_{n} \rightarrow q$. 
Proof: Since $X$ is reflexive and $\left\{x_{n}\right\}$ is bounded, $\omega_{w}\left(x_{n}\right)$ is nonempty. Noticing the weak lower semi-continuity of $\varphi(\cdot, u)$, we derive from condition (ii) that

$$
\varphi(v, u) \leqslant \varphi(q, u) \quad \forall v \in \omega_{w}\left(x_{n}\right) .
$$

However, since $\omega_{w}\left(x_{n}\right) \subset K$ and $q=P_{K} u$, we must have $v=q$ for all $v \in \omega_{w}\left(x_{n}\right)$. Thus $\omega_{w}\left(x_{n}\right)=\{q\}$ and $x_{n} \rightarrow q$.

To see $x_{n} \rightarrow q$, we observe that the inequality $\varphi\left(x_{n}, u\right) \leqslant \varphi(q, u)$ in condition (ii) is actually equivalent to the following one

$$
\left\|x_{n}\right\|^{2} \leqslant\|q\|^{2}+2\left\langle x_{n}-q, J(u)\right\rangle .
$$

Since $x_{n} \rightarrow q$, it follows that

$$
\underset{n}{\limsup }\left\|x_{n}\right\| \leqslant\|q\| .
$$

This and the uniform convexity of $X$ imply that $x_{n} \rightarrow q$.

\section{STRONG CONVERGENCE OF APPROXIMATING FIXED POINT SEQUENCES}

Let $C$ be a nonempty closed convex subset of a smooth and uniformly Banach space $X$ and let $T: C \rightarrow C$ be a nonexpansive mapping with a fixed point. Starting an arbitrary initial guess $x_{0}$, we can construct an approximating fixed point sequence of $T$ as follows. Take a sequence $\left\{t_{n}\right\}$ in $(0,1)$ so that $t_{n} \rightarrow 0$ as $n \rightarrow \infty$. Once $x_{n}$ has been constructed, we then construct two closed convex subsets $C_{n}$ and $Q_{n}$ such that

$$
C_{n}=\overline{c o}\left\{z \in C:\|z-T z\| \leqslant t_{n}\left\|x_{n}-T x_{n}\right\|\right\}
$$

and

$$
Q_{n}=\left\{v \in C:\left\langle x_{n}-v, J\left(x_{0}\right)-J\left(x_{n}\right)\right\rangle \geqslant 0\right\} .
$$

Then we define the $(n+1)$ th iterate $x_{n+1}$ to be the projection of $x_{0}$ onto the intersection of $C_{n}$ and $Q_{n}$ :

$$
x_{n+1}=P_{C_{n} \cap Q_{n}} x_{0} .
$$

Before discussing the convergence of the sequence $\left\{x_{n}\right\}$, we first use induction to verify that $\operatorname{Fix}(T) \subset C_{n} \cap Q_{n}$ and $x_{n+1}$ is well-defined. As a matter of fact, it is trivial that $\operatorname{Fix}(T) \subset C_{n}$ for all $n$. It is also trivial that $\operatorname{Fix}(T) \subset Q_{0}=C$ and thus $x_{1}=P_{C_{0} \cap Q_{0}} x_{0}$ is well-defined. Assume now $\operatorname{Fix}(T) \subset Q_{n}$ and $x_{n+1}$ is well-defined. We need to prove that $\operatorname{Fix}(T) \subset Q_{n+1}$ and $x_{n+2}$ is well-defined.

Since $x_{n+1}$ is the projection of $x_{0}$ onto $C_{n} \cap Q_{n}$, by Proposition 2.1 (ii) we have

$$
\left\langle x_{n+1}-z, J\left(x_{0}\right)-J\left(x_{n+1}\right)\right\rangle \geqslant 0 \quad \forall z \in C_{n} \cap Q_{n} .
$$


As $\operatorname{Fix}(T) \subset C_{n} \cap Q_{n}$, the last inequality holds, in particular, for all $z \in \operatorname{Fix}(T)$. This together with the definition of $Q_{n+1}$ implies that $\operatorname{Fix}(T) \subset Q_{n+1}$. Now as the projection of $x_{0}$ onto the nonempty closed convex subset $C_{n+1} \cap Q_{n+1}, x_{n+2}$ is well-defined.

We now state and prove the main result of this paper.

Theorem 3.1. Let $X$ be a real smooth and uniformly convex Banach space, $C$ a nonempty closed convex subset of $X$, and $T: C \rightarrow C$ a nonexpansive mapping such that $\operatorname{Fix}(T) \neq \emptyset$. Let $\left\{x_{n}\right\}$ be the sequence generated by the process (3.1). Then $\left\{x_{n}\right\}$ is an approximating fixed point sequence for $T$ and strongly convergent to a fixed point of $T$.

Proof: First we observe that $\left\{x_{n}\right\}$ is bounded. As a matter of fact, by the definition of $Q_{n}$, we have $x_{n}=P_{Q_{n}} x_{0}$. Hence by Proposition 2.1 (iii)

$$
\varphi\left(y, x_{n}\right)+\varphi\left(x_{n}, x_{0}\right) \leqslant \varphi\left(y, x_{0}\right) \quad \forall y \in Q_{n} .
$$

Since $\operatorname{Fix}(T) \subset Q_{n}$, we get

$$
\varphi\left(x_{n}, x_{0}\right) \leqslant \varphi\left(p, x_{0}\right) \quad \forall p \in \operatorname{Fix}(T) .
$$

This implies the boundedness of $\left\{x_{n}\right\}$. Because $x_{n+1}$ belongs to $Q_{n}$, we can substitute it for $y$ in (3.2) to get

$$
\varphi\left(x_{n+1}, x_{n}\right) \leqslant \varphi\left(x_{n+1}, x_{0}\right)-\varphi\left(x_{n}, x_{0}\right) .
$$

This implies that the real sequence $\left\{\varphi\left(x_{n}, x_{0}\right)\right\}$ is increasing (and also bounded) and thus $\lim _{n} \varphi\left(x_{n}, x_{0}\right)$ exists. Back to (3.4), we conclude that $\varphi\left(x_{n+1}, x_{n}\right) \rightarrow 0$ which implies $\left\|x_{n+1}-x_{n}\right\| \rightarrow 0$ by virtue of Proposition 2.1 (i).

We now claim that $\left\{x_{n}\right\}$ is an approximating fixed point sequence of $T$. Let $\widetilde{C}$ be a bounded closed convex subset of $C$ which contains all the points $x_{n}$ and $T x_{n}$ for all $n$ and let $\eta=\operatorname{diam}(\widetilde{C})$. Since $x_{n+1} \in C_{n}$ and by definition of $C_{n}$, we have

$$
\left\|x_{n+1}-\sum_{i=1}^{l} \lambda_{i} z_{i}\right\|<t_{n}
$$

where $\lambda_{i}>0$ satisfying $\sum_{i=1}^{l} \lambda_{i}=1$ and each $z_{i} \in C$ satisfies

$$
\left\|z_{i}-T z_{i}\right\| \leqslant t_{n}\left\|x_{n}-T x_{n}\right\| \leqslant \eta t_{n} .
$$

By Bruck [2], there exists a continuous strictly increasing function $\gamma$ (depending only on

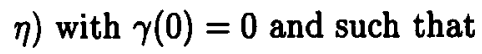

$$
\gamma\left(\left\|T\left(\sum_{i=1}^{m} \mu_{i} v_{i}\right)-\sum_{i=1}^{m} \mu_{i} T v_{i}\right\|\right) \leqslant \max \left(\left\|v_{i}-v_{j}\right\|-\left\|T v_{i}-T v_{j}\right\|: 1 \leqslant i, j \leqslant m\right)
$$


for all integers $m>1$, all points $\left\{v_{i}\right\}$ in $\widetilde{C}$, and all nonnegative numbers $\left\{\mu_{i}\right\}$ such that $\sum_{i=1}^{m} \mu_{i}=1$. It follows that

$$
\begin{aligned}
\left\|x_{n+1}-T x_{n+1}\right\| \leqslant & \left\|x_{n+1}-\sum_{i=1}^{l} \lambda_{i} z_{i}\right\|+\left\|\sum_{i=1}^{l} \lambda_{i}\left(z_{i}-T z_{i}\right)\right\| \\
& +\left\|\sum_{i=1}^{l} \lambda_{i} T z_{i}-T\left(\sum_{i=1}^{l} \lambda_{i} z_{i}\right)\right\|+\left\|T\left(\sum_{i=1}^{l} \lambda_{i} z_{i}\right)-T x_{n+1}\right\| \\
& \leqslant(2+\eta) t_{n}+\gamma^{-1}\left(\max \left(\left\|z_{i}-z_{j}\right\|-\left\|T z_{i}-T z_{j}\right\|: 1 \leqslant i, j \leqslant l\right)\right) \\
& \leqslant(2+\eta) t_{n}+\gamma^{-1}\left(\max \left(\left\|z_{i}-T z_{i}\right\|+\left\|z_{j}-T z_{j}\right\|: 1 \leqslant i, j \leqslant l\right)\right) \\
& \leqslant(2+\eta) t_{n}+\gamma^{-1}\left(2 \eta t_{n}\right) \rightarrow 0 .
\end{aligned}
$$

Therefore, $\left\{x_{n}\right\}$ is an approximating fixed point sequence.

Finally let us prove that $\left\{x_{n}\right\}$ is strongly convergent to a fixed point of $T$. By the demiclosedness principle (Lemma 1.1), we have $\omega_{w}\left(x_{n}\right) \subset \operatorname{Fix}(T)$. Let $q=P_{\mathrm{Fix}(T)} x_{0}$. By (3.3) we see that $\varphi\left(x_{n}, x_{0}\right) \leqslant \varphi\left(q, x_{0}\right)$ for all $n$. Therefore, applying Lemma 2.2 to the nonempty closed convex subset $K:=\mathrm{Fix}(T)$, we conclude that $x_{n} \rightarrow q$.

\section{REFERENCES}

[1] Y.I. Alber and S. Guerre-Delabriere, 'On the projection methods for fixed point problems', Analysis (Munich) 21 (2001), 17-39.

[2] R.E. Bruck, 'On the convex approximation property and the asymptotic behaviour of nonlinear contractions in Banach spaces', Israel J. Math. 38 (1981), 304-314.

[3] A. Genel and J. Lindenstrass, 'An example concerning fixed points', Israel J. Math. 22 (1975), 81-86.

[4] K. Goebel and W.A. Kirk, Topics in metric fixed point theory, Cambridge Studies in Advanced Mathematics 28 (Cambridge University Press, Cambridge, 1990).

[5] S. Kamimura and W. Takahashi, 'Strong convergence of a proximal-type algorithm in a Banach space', SIAM J. Optim. 13 (2003), 938-945.

[6] T.H. Kim and H.K. Xu, 'Strong convergence of modified Mann iterations', Nonlinear Anal. 61 (2005), 51-60.

[7] T.H. Kim and H.K. Xu, 'Strong convergence of modified Mann iterations for asymptotically nonexpansive mappings and semigroups', Nonlinear Anal. 64 (2006), 1140-1152.

[8] W.R. Mann, 'Mean value methods in iteration', Proc. Amer. Math. Soc. 4 (1953), 506-510.

[9] G. Marino and H.K. Xu, 'Convergence of generalized proximal point algorithms', Commun. Pure App. Anal. 3 (2004), 791-808.

[10] C. Matinez-Yanes and H.K. Xu, 'Strong convergence of the CQ method for fixed point processes', Nonlinear Anal. 64 (2006), 2400-2411. 
[11] K. Nakajo and W. Takahashi, 'Strong convergence theorems for nonexpansive mappings and nonexpansive semigroups', J. Math. Anal. Appl. 279 (2003), 372-379.

[12] S. Reich, 'Weak convergence theorems for nonexpansive mappings in Banach spaces', $J$. Math. Anal. Appl. 67 (1979), 274-276.

[13] N. Shioji and W. Takahashi, 'Strong convergence of approximated sequences for nonexpansive mappings in Banach spaces', Proc. Amer. Math. Soc. 125 (1997), 3641-3645.

[14] M.V. Solodov and B.F. Svaiter, 'Forcing strong convergence of proximal point iterations in a Hilbert space', Math. Program. Ser. A 87 (2000), 189-202.

[15] R. Wittmann, 'Approximation of fixed points of nonexpansive mappings', Arch. Math. (Basel) 58 (1992), 486-491.

[16] H.K. X, 'Inequalities in Banach spaces with applications', Nonlinear Anal. 16 (1991), 1127-1138.

[17] H.K. Xu, 'Iterative algorithms for nonlinear operators', J. London Math. Soc. (2) 66 (2002), 240-256.

School of Mathematical Sciences

University of KwaZulu-Natal

Westville Campus

Private Bag X54001

Durban 4000

South Africa

e-mail: xuhk@ukzn.ac.za 